\title{
Quantitative Mixing Results and Inner Functions
}

\author{
J.L. Fernández* \\ M.V. Melián* \\ Universidad Autónoma de Madrid \\ Madrid, Spain \\ Universidad Autónoma de Madrid \\ Madrid, Spain \\ joseluis.fernandez@uam.es \\ mavi.melian@uam.es \\ D. Pestana ${ }^{\dagger}$ \\ Universidad Carlos III de Madrid \\ Madrid, Spain \\ domingo.pestana@uc3m.es
}

\begin{abstract}
We study in this paper estimates on the size of the sets of points which are well approximated by orbits of other points under certain dynamical systems. We apply the results obtained to the particular case of the dynamical system generated by inner functions in the unit disk of the complex plane.
\end{abstract}

AMS Subject Classification (2000): 30D05, 30D50, 37A05, 37A25, 37F10, 28D05, 11K55.

*Research supported by Grant BFM2003-04780 from Ministerio de Ciencia y Tecnología, Spain

${ }^{\dagger}$ Research supported by Grants BFM2003-04780 and BFM-2003-06335-C03-02, Ministerio de Ciencia y Tecnología, Spain 


\section{Introduction}

If a measure preserving transformation $T$ is mixing, then pre-images under $T$ distribute themselves somehow regularly along the base space. We are interested in this paper in quantifying this regularity.

A close analogue and a source of inspiration for this paper is furnished by Poincaré's recurrence theorem and certain quantitative refinements due to M. Boshernitzan. But the main motivation for this work has been the study of inner functions as dynamical systems and they provide concrete examples of applications of our results.

\section{Quantitative Mixing Results.}

Throughout this paper $(X, d)$ will be a metric space endowed with a finite measure $\mu$ over the Borel sets and such that the support of $\mu$ is equal to $X$. As a normalization we shall assume that $\mu(X)=1$. Also $T: X \longrightarrow X$ will be a measure preserving transformation, i.e. a measure such that $\mu\left(T^{-1}(A)\right)=\mu(A)$ for all Borel set $A$.

The classical recurrence theorem of Poincaré (see for example [F, p.61]) says that

Theorem A (H. Poincaré). If $X$ is separable, then $\mu$-almost every point of $X$ is recurrent in the sense that

$$
\liminf _{n \rightarrow \infty} d\left(T^{n}(x), x\right)=0 .
$$

Here $T^{n}$ denotes the $n$-th fold composition $T^{n}=T \circ T \circ \cdots \circ T$. It is natural to ask if the orbit $\left\{T^{n}(x)\right\}$ of the point $x$ not only comes back to any neighborhood of $x$ itself as Poincaré's Theorem asserts, but whether it also visits any neighborhood of a previously chosen point $x_{0} \in X$. The main aim of this paper is to obtain results in this direction.

Under the additional hypothesis of ergodicity one can obtain the following result which parallels Theorem A. We recall that the transformation $T$ is ergodic if the only $T$-invariant sets (up to sets of $\mu$-measure zero) are trivial, i.e. they have zero $\mu$-measure or their complements have zero $\mu$-measure.

Theorem A'. If $T: X \longrightarrow X$ is ergodic then, for any $x_{0} \in X$, we have that

$$
\liminf _{n \rightarrow \infty} d\left(T^{n}(x), x_{0}\right)=0
$$

for $\mu$-almost all $x \in X$.

For the sake of completeness we will include a proof of Theorem A' on Section 2.

M. Boshernitzan obtained in [B] the following quantitative version of Theorem A.

Theorem B (M. Boshernitzan). If $X$ is separable and the Hausdorff $\alpha$-measure $H_{\alpha}$ is $\sigma$-finite on $X$ for some $\alpha>0$, then for $\mu$-almost all $x \in X$,

$$
\liminf _{n \rightarrow \infty} n^{1 / \alpha} d\left(T^{n}(x), x\right)<\infty .
$$

M. Boshernitzan also proved that if $H_{\alpha}(X)=0$, then for $\mu$-almost all $x \in X$,

$$
\liminf _{n \rightarrow \infty} n^{1 / \alpha} d\left(T^{n}(x), x\right)=0
$$


and when the measure $\mu$ agrees with $H_{\alpha}$ for some $\alpha>0$, then for $\mu$-almost all $x \in X$,

$$
\liminf _{n \rightarrow \infty} n^{1 / \alpha} d\left(T^{n}(x), x\right) \leq 1 .
$$

Recently, L. Barreira and B. Saussol [BS] have reformulated the result (1) in terms of the return time of a point $x \in X$ into the ball $B(x, r)$. They have also obtained a generalization of (1) for subsets of $\mathbf{R}^{N}$ in terms of the lower pointwise dimension of $\mu$ at the point $x \in X$ instead of the Hausdorff measure of $X$. We recall that the lower and upper pointwise dimension at $x$ are defined, respectively, as

$$
\underline{d}_{\mu}(x)=\liminf _{r \rightarrow 0} \frac{\log \mu(B(x, r))}{\log r} \quad \text { and } \quad \bar{d}_{\mu}(x)=\limsup _{r \rightarrow 0} \frac{\log \mu(B(x, r))}{\log r} .
$$

Theorem C (L. Barreira and B. Saussol). Let us suppose that $X \subset \mathbf{R}^{N}$ for some $N \in \mathbf{N}$. Then

$$
\liminf _{n \rightarrow \infty} n^{1 / \alpha} d\left(T^{n}(x), x\right)=0,
$$

for $\mu$-almost every $x \in X$ such that $\underline{d}_{\mu}(x)<\alpha$.

More recently, some new quantitative recurrence results along these lines have been obtained by several authors relating various recurrence indicators with entropy and dimension, see e.g. [ACS], [BGI], [G] and [STV].

In order to obtain a quantitative version of Theorem A' we will need the additional hypothesis that $T$ is uniformly mixing at $x_{0}$ (see Section 3 for the definition).

Theorem 1. Let $\left\{r_{n}\right\}$ be a decreasing sequence of positive numbers tending to zero as $n \rightarrow \infty$. If $T$ is uniformly mixing at a point $x_{0} \in X$ and

$$
\sum_{n=1}^{\infty} \mu\left(B\left(x_{0}, r_{n}\right)\right)=\infty
$$

then

$$
\lim _{n \rightarrow \infty} \frac{\#\left\{i \leq n: d\left(T^{i}(x), x_{0}\right) \leq r_{i}\right\}}{\sum_{j=1}^{n} \mu\left(B\left(x_{0}, r_{j}\right)\right)}=1, \quad \text { for } \mu \text {-almost every } x \in X
$$

In particular

$$
\liminf _{n \rightarrow \infty} \frac{d\left(T^{n}(x), x_{0}\right)}{r_{n}} \leq 1, \quad \text { for } \mu \text {-almost every } x \in X
$$

The notation \#A means the number of elements of the set $A$.

As a complement of this statement observe that if the sequence $\left\{r_{n}\right\}$ goes fast enough to zero in such a way that

$$
\sum_{n=1}^{\infty} \mu\left(B\left(x_{0}, r_{n}\right)\right)<\infty,
$$

then, as a consequence of Borel-Cantelli lemma it is easy to see (see Proposition 1 in Section 3) that 


$$
\liminf _{n \rightarrow \infty} \frac{d\left(T^{n}(x), x_{0}\right)}{r_{n}} \geq 1, \quad \text { for } \mu \text {-almost every } x \in X .
$$

As a consequence of Theorem 1 we obtain the following result which we can compare with Theorems B and C.

Corollary 1. If $T$ is uniformly mixing at a point $x_{0} \in X$ and $\alpha>\bar{d}_{\mu}\left(x_{0}\right)$, then

$$
\liminf _{n \rightarrow \infty} n^{1 / \alpha} d\left(T^{n}(x), x_{0}\right)=0, \quad \text { for } \mu \text {-almost every } x \in X .
$$

As a consequence of (2) we also get that if $\alpha<\underline{d}_{\mu}\left(x_{0}\right)$, then

$$
\liminf _{n \rightarrow \infty} n^{1 / \alpha} d\left(T^{n}(x), x_{0}\right)=\infty, \quad \text { for } \mu \text {-almost every } x \in X .
$$

\section{Inner Functions.}

The main motivation of this work has been the study of mixing properties of inner functions. We recall that the classical Fatou's Theorem asserts that a bounded holomorphic function $f: \mathbf{D} \longrightarrow \mathbf{C}$, from the unit disk $\mathbf{D}$ into the complex plane $\mathbf{C}$, has radial limits almost everywhere. A holomorphic function $f$ defined on $\mathbf{D}$ and with values in $\mathbf{D}$ is called an inner function if the radial limits

$$
f^{*}(\xi):=\lim _{r \rightarrow 1^{-}} f(r \xi)
$$

(which exists for almost every $\xi$ by Fatou's Theorem) have modulus 1 for almost every $\xi \in \partial \mathbf{D}$. Here and hereafter $\partial \mathbf{D}$ will denote the boundary of the unit disk $\mathbf{D}$. Therefore if $f$ is inner, the radial limits (3) define a mapping $f^{*}: \partial \mathbf{D} \longrightarrow \partial \mathbf{D}$ up to a set of zero Lebesgue measure. The fact that $f$ is holomorphic implies the following well known result, see e.g. $[R]$.

Theorem D (Löwner's lemma) If $f: \mathbf{D} \longrightarrow \mathbf{D}$ is an inner function then $f^{*}: \partial \mathbf{D} \longrightarrow$ $\partial \mathbf{D}$ preserves Lebesgue measure if and only if $f(0)=0$.

Every inner function has a representation of the form

$$
f(z)=e^{i \theta} \prod_{j} \frac{\left|a_{j}\right|}{a_{j}} \frac{z-a_{j}}{1-\overline{a_{j}} z} \exp \left(-\int_{\partial \mathbf{D}} \frac{\xi+z}{\xi-z} d \nu(\xi)\right)
$$

where $\left\{a_{j}\right\}$ is the sequence of the zeroes of $f$ in $\mathbf{D}$ (possibly empty) and $\nu$ is a singular non negative measure in $\partial \mathbf{D}$. The dynamics of an inner function in $\partial \mathbf{D}$ can be very complicated. In fact, $f^{*}: \partial \mathbf{D} \longrightarrow \partial \mathbf{D}$ can be very discontinuous. If $z \in \partial \mathbf{D}$ is a singular point of $f$, i.e. if $z$ is an accumulation point of the sequence $\left\{a_{j}\right\}$ of zeroes or if $z$ belongs to the support of the singular measure $\nu$, then $f^{*}: \partial \mathbf{D} \longrightarrow \partial \mathbf{D}$ maps every neighborhood of $z$ onto the whole of $\partial \mathbf{D}$. On the other hand, if $z \in \partial \mathbf{D}$ is not a singular point, then $f$ extends holomorphically to a neighborhood of $z$.

It is remarkable that, in spite of the fact that $f^{*}$ is only defined up to a set of measure zero, one can prove results about the behaviour of $f^{*}$ on sets of zero Lebesgue measure. 
For example, in $[\mathrm{FP}]$ (see also $[\mathrm{FPR}]$ and $[\mathrm{PR}]$ for extensions of these results) it was proved that for any Borel subset $A$ of $\partial \mathbf{D}$

$$
\operatorname{cap}_{\alpha}\left(\left(f^{*}\right)^{-1}(A)\right) \geq \operatorname{cap}_{\alpha}(A),
$$

where $\operatorname{cap}_{\alpha}$ denotes Riesz $\alpha$-capacity for $0<\alpha<1$ and logarithmic capacity for $\alpha=0$. Therefore, it follows that

$$
\operatorname{Dim}\left(\left(f^{*}\right)^{-1}(A)\right) \geq \operatorname{Dim}(A),
$$

where Dim denotes Hausdorff dimension.

If $f$ is inner with a fixed point in $\mathbf{D}$, but it is not conjugated to a rotation, J. Aaronson $[\mathrm{A}]$ and J.H. Neuwirth [N] proved, independently, that $f^{*}$ is exact with respect to harmonic measure and therefore mixing and ergodic. In fact, inner functions are also ergodic with respect to $\alpha$-capacity [FPR]. An interesting study of some dynamical properties of inner functions is contained in the works of M. Craizer. In [C1] he proves that if $f^{\prime}$ belongs to the Nevanlinna class, then the measure theoretic entropy of $f^{*}$ is finite and it can be calculated by the formula

$$
h\left(f^{*}\right)=\frac{1}{2 \pi} \int_{0}^{2 \pi} \log \left|\left(f^{*}\right)^{\prime}(x)\right| d x,
$$

where $\left(f^{*}\right)^{\prime}$ denotes the angular derivative of $f$. He also proves that the extension $f^{*}$ of an inner function with a fixed point in $\mathbf{D}$ is equivalent to a generalized Bernoulli shift, see $[\mathrm{C} 2]$.

The mixing properties of inner functions are even stronger. In this sense Ch. Pommerenke $[\mathrm{P}]$ has shown the following

Theorem E (Ch. Pommerenke). Let $f: \mathbf{D} \longrightarrow \mathbf{D}$ be an inner function with $f(0)=0$, but not a rotation. Then, there exists a positive absolute constant $K$ such that

$$
\left|\frac{m\left[B \cap\left(f^{*}\right)^{-n}(A)\right]}{m(A)}-m(B)\right| \leq K e^{-\alpha n},
$$

for all $n \in \mathbf{N}$, for all arcs $A, B \subset \partial \mathbf{D}$, where $\alpha=\max \left\{1 / 2,\left|f^{\prime}(0)\right|\right\}$.

Here $m(\cdot)$ denotes normalized Lebesgue measure. Notice that once you have this result for all $\operatorname{arcs} A, B$, one can automatically obtain the same conclusion for all $\operatorname{arcs} B$ and all Borel sets $A$ with $m(A)>0$.

The mixing property (4) of inner functions allows us to prove the following result.

Theorem 2. Let $f: \mathbf{D} \longrightarrow \mathbf{D}$ be an inner function with $f(0)=0$, but not a rotation. Let $\xi_{0}$ be a point in $\partial \mathbf{D}$ and let $\left\{r_{n}\right\}$ be a decreasing sequence of positive numbers. If $\sum_{n=1}^{\infty} r_{n}=\infty$, then

$$
\lim _{N \rightarrow \infty} \frac{\#\left\{n \leq N: d\left(\left(f^{*}\right)^{n}(\xi), \xi_{0}\right)<r_{n}\right\}}{\sum_{n=1}^{N} r_{n}}=1, \quad \text { for almost every } \xi \in \partial \mathbf{D} .
$$

In particular,

$$
\liminf _{n \rightarrow \infty} \frac{d\left(\left(f^{*}\right)^{n}(\xi), \xi_{0}\right)}{r_{n}} \leq 1, \quad \text { for almost every } \xi \in \partial \mathbf{D}
$$


Here $d$ denotes the angular distance in $\partial \mathbf{D}$. Observe that in particular we get that

$$
\lim _{N \rightarrow \infty} \frac{\#\left\{n \leq N: d\left(\left(f^{*}\right)^{n}(\xi), \xi_{0}\right)<1 / n\right\}}{\log N}=1, \quad \text { for almost every } \xi \in \partial \mathbf{D} .
$$

As a consequence of (2) one has also that if $r_{n}$ tends fast enough to zero, i.e. if $\sum_{n=1}^{\infty} r_{n}<\infty$, then

$$
\liminf _{n \rightarrow \infty} \frac{d\left(\left(f^{*}\right)^{n}(\xi), \xi_{0}\right)}{r_{n}} \geq 1, \quad \text { for almost every } \xi \in \partial \mathbf{D} .
$$

A stronger result about inner functions with a fixed point $p \in \mathbf{D}$ will be obtained in Section 4 .

The outline of the paper is as follows. In Section 2 we will prove Theorem A'. In Section 3 we give the definition of uniform mixing and prove Theorem 1 . Section 4 contains the results about inner functions. Finally, Section 5 contains an application to the transformation in $[0,1]$ given by $x \mapsto m x(\bmod 1)$ where $m \in \mathbf{N}$.

Before closing this introduction we would like to thank the referee for some useful suggestions.

\section{A mixing Poincaré-type Theorem}

In this section we will prove Theorem A', a result which parallels Poincaré Recurrence Theorem. First of all we begin with a definition.

Definition 1. A dynamical metric system $(X, d, \mathcal{A}, \mu, T)$ is a dynamical system with a compatible metric d. In other words, $(X, d)$ is a metric space, $\mathcal{A}$ is the $\sigma$-algebra of the Borel sets of $(X, d), \mu$ is a probability Borel measure in $X$ such that $\operatorname{supp}(\mu)=X$, and $T: X \longrightarrow X$ is a preserving measure transformation in $X$, i.e.,

$$
\mu\left(T^{-1}(A)\right)=\mu(A), \quad \text { for all } A \in \mathcal{A} .
$$

Since the intersection of a numerable collection of full measure sets has also full measure, Theorem A' can be stated in the following equivalent way:

Theorem A'. Let $(X, d, \mathcal{A}, \mu, T)$ be an ergodic dynamical metric system. Given a sequence $\left\{x_{i}\right\}_{i=1}^{\infty} \subset X$, then

$$
\liminf _{n \rightarrow \infty} d\left(T^{n}(x), x_{i}\right)=0, \quad \text { for } i=1,2, \ldots
$$

for $\mu$-almost all $x \in X$.

Proof. It is enough to prove the result for a unique point $x_{0} \in X$. First of all we recall that the ergodicity of $T$ implies that if $A, B$ have positive $\mu$-measure then $A \cap T^{-k}(B)$ has also positive $\mu$-measure for some $k \in \mathbf{N}$ (see [W, p.27]). Let us define the sets

$$
X_{n}=X \backslash\left(\bigcup_{j=1}^{\infty} T^{-j}\left(B\left(x_{0}, 1 / n\right)\right)\right) .
$$


Notice that

$$
\liminf _{m \rightarrow \infty} d\left(T^{m}(x), x_{0}\right)>0 \quad \text { if and only if } \quad x \in \bigcup_{n=1}^{\infty} X_{n} .
$$

Moreover, for all $k \in \mathbf{N}$

$$
X_{n} \cap T^{-k}\left(B\left(x_{0}, 1 / n\right)\right)=\emptyset .
$$

Since $B\left(x_{0}, 1 / n\right)$ has positive $\mu$-measure (because we are assuming that $X=\operatorname{supp} \mu$ ), by ergodicity, we have that $\mu\left(X_{n}\right)=0$ for all $n$ and so $\mu\left(\cup X_{n}\right)=0$.

\section{Quantitative mixing results}

Once again, let $(X, d, \mathcal{A}, \mu, T)$ be a dynamical metric system. In this section we study the size of the set

$$
\mathcal{H}\left(x_{0}\right)=\left\{x \in X: d\left(T^{n}(x), x_{0}\right)<r_{n} \text { for infinitely many } n\right\}
$$

where $\left\{r_{n}\right\}$ is a given sequence of positive numbers and $x_{0}$ is an arbitrary point in $X$. Observe that $\mathcal{H}\left(x_{0}\right)$ also depends on the sequence $\left\{r_{n}\right\}$. If the sequence $r_{n}$ is constant, $\mathcal{H}\left(x_{0}\right)$ is $T$-invariant, but, in general, this is not the case.

Let us denote $B_{k}=B\left(x_{0}, r_{k}\right)$ and $A_{k}=T^{-k}\left(B_{k}\right)$. With these notations, we have that

$$
\mathcal{H}\left(x_{0}\right)=\left\{x \in X: x \in A_{n} \text { for infinitely many } n\right\}=\bigcap_{k=1}^{\infty} \bigcup_{n=k}^{\infty} A_{n} .
$$

The following result on the size of the set $\mathcal{H}\left(x_{0}\right)$ is an easy consequence of the direct part of Borel-Cantelli lemma.

Proposition 1. Let $(X, d, \mathcal{A}, \mu, T)$ be a dynamical metric system and let $\left\{r_{n}\right\}$ be a sequence of positive numbers. Let $x_{0} \in X$,

$$
\text { if } \quad \sum_{n=1}^{\infty} \mu\left(B\left(x_{0}, r_{n}\right)\right)<\infty \quad \text { then } \quad \mu\left(\mathcal{H}\left(x_{0}\right)\right)=0 .
$$

Proof. Since $T$ preserves measure, we have that $\mu\left(B_{k}\right)=\mu\left(A_{k}\right)$ for all $k \in \mathbf{N}$ and therefore

$$
\sum_{n=1}^{\infty} \mu\left(A_{n}\right)=\sum_{n=1}^{\infty} \mu\left(B_{n}\right)<\infty
$$

From Borel-Cantelli lemma it follows that $\mu\left(\mathcal{H}\left(x_{0}\right)\right)=0$.

Corollary 2. If $\sum_{n=1}^{\infty} \mu\left(B\left(x_{0}, r_{n}\right)\right)<\infty$ then

$$
\liminf _{n \rightarrow \infty} \frac{d\left(T^{n}(x), x_{0}\right)}{r_{n}} \geq 1, \quad \text { for } \mu \text {-almost every } x \in X \text {. }
$$

We give now a definition that we will need in the sequel. 
Definition 2. Let $(X, d, \mathcal{A}, \mu, T)$ be a dynamical metric system. We will say that the transformation $T$ is uniformly mixing at the point $x_{0} \in X$ if there exists a positive decreasing continuous function $\Phi:[1, \infty) \longrightarrow \mathbf{R}$ such that

$$
\int_{1}^{\infty} \Phi(x) d x<\infty
$$

and

$$
\left|\frac{\mu\left[B_{1} \cap T^{-n}\left(B_{2}\right)\right]}{\mu\left(B_{2}\right)}-\mu\left(B_{1}\right)\right| \leq \Phi(n),
$$

for all $n \in \mathbf{N}$ and for all pair of balls $B_{1}, B_{2}$ centered at $x_{0}$.

A more precise statement of Theorem 1 is the following:

Theorem 1. Let $(X, d, \mathcal{A}, \mu, T)$ be a dynamical metric system. Let $x_{0} \in X$ and let $\left\{r_{n}\right\}$ be a decreasing sequence of positive numbers. Define the set $\mathcal{H}\left(x_{0}\right)$ by (5). If $T$ is uniformly mixing at $x_{0}$ and

$$
\sum_{n=1}^{\infty} \mu\left(B\left(x_{0}, r_{n}\right)\right)=\infty, \quad \text { then } \quad \mu\left(\mathcal{H}\left(x_{0}\right)\right)=1
$$

In fact, one can obtain the following quantitative version

$$
\lim _{n \rightarrow \infty} \frac{\#\left\{i \leq n: d\left(T^{i}(x), x_{0}\right) \leq r_{i}\right\}}{\sum_{j=1}^{n} \mu\left(B\left(x_{0}, r_{j}\right)\right)}=1, \quad \text { for } \mu \text {-almost every } x \in X .
$$

In particular

$$
\liminf _{n \rightarrow \infty} \frac{d\left(T^{n}(x), x_{0}\right)}{r_{n}} \leq 1, \quad \text { for } \mu \text {-almost every } x \in X
$$

Our argument follows the line of proof of strong laws of large numbers with PaleyZygmund inequality providing the key estimate. A nice exposition of this kind of arguments, in a closely related context, may be found in $[\mathrm{K}]$. The relevant fact here is that uniform mixing implies that a certain family of sets is independent in a precise asymptotical sense. The related assumption of quasi-independence would allow us to prove, following the argument in $[\mathrm{S}]$, for instance, that

$$
\mu\left[\left\{x \in X: \limsup _{n \rightarrow \infty} \frac{\#\left\{i \leq n: d\left(T^{i}(x), x_{0}\right) \leq r_{i}\right\}}{\sum_{j=1}^{n} \mu\left(B\left(x_{0}, r_{j}\right)\right)}>0\right\}\right]>0 .
$$

But, in general, the set above is not $T$-invariant and therefore one can not readily conclude that it has full measure.

Lemma (Payley-Zygmund Inequality). Let $(X, \mathcal{A}, \mu)$ be a probability space and let $Z: X \longrightarrow \mathbf{R}$ be a positive random variable. Then, for $0<\lambda<1$,

$$
\mu[\{x \in X: Z(x)>\lambda \mathbf{E}(Z)\}] \geq(1-\lambda)^{2} \frac{\mathbf{E}(Z)^{2}}{\mathbf{E}\left(Z^{2}\right)} .
$$


Proof. We will denote by $\chi_{A}$ the indicator function of $A$. From Cauchy-Schwarz inequality we deduce that

$$
\mathbf{E}(Z)=\mathbf{E}\left(Z \chi_{\{Z \leq \lambda \mathbf{E}(Z)\}}\right)+\mathbf{E}\left(Z \chi_{\{Z>\lambda \mathbf{E}(Z)\}}\right) \leq \lambda \mathbf{E}(Z)+\mathbf{E}\left(Z^{2}\right)^{1 / 2} \mu[Z>\lambda \mathbf{E}(Z)]^{1 / 2},
$$

and the result follows.

Proof of Theorem 1. Let us denote $B_{k}=B\left(x_{0}, r_{k}\right)$ and $A_{k}=T^{-k}\left(B_{k}\right)$. Recall that

$$
\mathcal{H}\left(x_{0}\right)=\left\{x \in X: x \in A_{n} \text { for infinitely many } n\right\}
$$

and that, since $T$ preserves measure, we have that $\mu\left(B_{k}\right)=\mu\left(A_{k}\right)$ for all $k \in \mathbf{N}$.

If $j=k+\ell, \ell \geq 0$, we have that

$$
\begin{aligned}
\mu\left(A_{k} \cap A_{j}\right) & =\mu\left(T^{-k}\left(B_{k}\right) \cap T^{-k}\left(T^{-\ell}\left(B_{j}\right)\right)\right) \\
& =\mu\left(T^{-k}\left(B_{k} \cap T^{-\ell}\left(B_{j}\right)\right)\right) \\
& =\mu\left(B_{k} \cap T^{-\ell}\left(B_{j}\right)\right),
\end{aligned}
$$

using again that $T$ preserves the measure $\mu$. But since $T$ is uniformly mixing at $x_{0}$ we get from (7) that

$$
\begin{aligned}
\mu\left(A_{k} \cap A_{j}\right) & \leq \mu\left(B_{k}\right) \mu\left(B_{j}\right)+\Phi(\ell) \mu\left(B_{j}\right) \\
& =\mu\left(A_{k}\right) \mu\left(A_{j}\right)+\Phi(\ell) \mu\left(A_{j}\right),
\end{aligned}
$$

where $\Phi$ is the function given in Definition 2.

Let us denote by $Z_{n}$ and $Z$ the counting functions

$$
Z_{n}=\sum_{k=1}^{n} \chi_{A_{k}} \quad \text { and } \quad Z=\sum_{k=1}^{\infty} \chi_{A_{k}},
$$

where $\chi_{A_{k}}$ is the indicator function of $A_{k}$. Observe that $\mathcal{H}\left(x_{0}\right)=\{x \in X: Z(x)=\infty\}$.

Expanding $Z_{n}^{2}$ we get

$$
\mathbf{E}\left(Z_{n}^{2}\right)=\mathbf{E}\left[\sum_{k=1}^{n} \chi_{A_{k}}+\sum_{\substack{k, j=1 \\ k \neq j}}^{n} \chi_{A_{k} \cap A_{j}}\right]=\sum_{k=1}^{n} \mu\left(A_{k}\right)+2 \sum_{\substack{k, j=1 \\ k<j}}^{n} \mu\left(A_{k} \cap A_{j}\right)
$$

and then using (8) we deduce

$$
\begin{aligned}
\mathbf{E}\left(Z_{n}^{2}\right) & \leq \sum_{k=1}^{n} \mu\left(A_{k}\right)+2 \sum_{\substack{k, j=1 \\
k<j}}^{n} \mu\left(A_{k}\right) \mu\left(A_{j}\right)+2 \sum_{\substack{k, j=1 \\
k<j}}^{n} \Phi(j-k) \mu\left(A_{j}\right) \\
& \leq \mathbf{E}\left(Z_{n}\right)+\mathbf{E}\left(Z_{n}\right)^{2}+2 \sum_{\substack{k, j=1 \\
k<j}}^{n} \Phi(j-k) \mu\left(A_{j}\right) .
\end{aligned}
$$

But $\mu\left(A_{n}\right)=\mu\left(B_{n}\right)$ decreases as $n \rightarrow \infty$ because $\left\{r_{n}\right\}$ is decreasing. Therefore

$$
\begin{aligned}
\mathbf{E}\left(Z_{n}^{2}\right) & \leq \mathbf{E}\left(Z_{n}\right)+\mathbf{E}\left(Z_{n}\right)^{2}+2 \sum_{k=1}^{n} \mu\left(A_{k}\right) \sum_{j=k+1}^{n} \Phi(j-k) \\
& \leq\left(1+2 \sum_{\ell=1}^{\infty} \Phi(\ell)\right) \mathbf{E}\left(Z_{n}\right)+\mathbf{E}\left(Z_{n}\right)^{2} .
\end{aligned}
$$


Notice that $\sum_{\ell=1}^{\infty} \Phi(\ell)$ is finite because of (6). By applying Paley-Zygmund Lemma we obtain from (9) that

$$
\begin{aligned}
\mu\left[\left\{x \in X: Z(x)>\lambda \mathbf{E}\left(Z_{n}\right)\right\}\right] & \geq \mu\left[\left\{x \in X: Z_{n}(x)>\lambda \mathbf{E}\left(Z_{n}\right)\right\}\right] \\
& \geq(1-\lambda)^{2} \frac{\mathbf{E}\left(Z_{n}\right)}{1+2 \sum_{\ell=1}^{\infty} \Phi(\ell)+\mathbf{E}\left(Z_{n}\right)} .
\end{aligned}
$$

Since

$$
\mathbf{E}\left(Z_{n}\right)=\sum_{k=1}^{n} \mu\left(A_{k}\right)=\sum_{k=1}^{n} \mu\left(B\left(x_{0}, r_{k}\right)\right)
$$

we deduce, from the hypothesis of the theorem, that $\mathbf{E}\left(Z_{n}\right) \rightarrow \infty$ as $n \rightarrow \infty$. Therefore, we get from (10) that

$$
\mu[\{x \in X: Z(x)=\infty\}] \geq(1-\lambda)^{2}, \quad \text { for } 0<\lambda<1 .
$$

As a consequence we conclude that $\mathcal{H}\left(x_{0}\right)$ has full $\mu$-measure.

To prove the quantitative version, let us observe that from (9) it follows that

$$
\mathbf{E}\left[\left(Z_{n}-\mathbf{E}\left(Z_{n}\right)\right)^{2}\right] \leq\left(1+2 \sum_{\ell=1}^{\infty} \Phi(\ell)\right) \mathbf{E}\left(Z_{n}\right) .
$$

Therefore, the random variable $Y_{n}=Z_{n} / \mathbf{E}\left(Z_{n}\right)-1=\left(Z_{n}-\mathbf{E}\left(Z_{n}\right)\right) / \mathbf{E}\left(Z_{n}\right)$ verifies that

$$
\mathbf{E}\left(Y_{n}^{2}\right) \leq \frac{C}{\mathbf{E}\left(Z_{n}\right)} .
$$

Since, by hypothesis, $\mathbf{E}\left(Z_{n}\right) \rightarrow \infty$ as $n \rightarrow \infty$ we can define the following sequence $\left\{n_{k}\right\}$ of natural numbers:

$$
n_{k}:=\inf \left\{n \in \mathbf{N}: \mathbf{E}\left(Z_{n}\right) \geq k^{2}\right\} .
$$

Then, using (11), we have that

$$
\sum_{k=1}^{\infty} \mathbf{E}\left(Y_{n_{k}}^{2}\right) \leq \sum_{k=1}^{\infty} \frac{C}{k^{2}}<\infty
$$

and therefore $\mathbf{E}\left[\sum_{k=1}^{\infty} Y_{n_{k}}^{2}\right]<\infty$. Hence $\sum_{k=1}^{\infty} Y_{n_{k}}^{2}<\infty$ in a subset of $X$ with full $\mu$-measure. From this fact it follows that $Y_{n_{k}} \rightarrow 0$ in that set, or equivalently,

$$
\frac{Z_{n_{k}}}{\mathbf{E}\left(Z_{n_{k}}\right)} \rightarrow 1, \quad \mu \text {-almost everywhere, }
$$

as $k \rightarrow \infty$. Finally, if $n_{k} \leq n<n_{k+1}$, we have that

$$
\frac{Z_{n}}{\mathbf{E}\left(Z_{n}\right)} \leq \frac{Z_{n_{k+1}}}{\mathbf{E}\left(Z_{n_{k}}\right)}=\frac{Z_{n_{k+1}}}{\mathbf{E}\left(Z_{n_{k+1}}\right)} \frac{\mathbf{E}\left(Z_{n_{k+1}}\right)}{\mathbf{E}\left(Z_{n_{k}}\right)} \leq \frac{Z_{n_{k+1}}}{\mathbf{E}\left(Z_{n_{k+1}}\right)} \frac{(k+2)^{2}}{k^{2}}
$$

and

$$
\frac{Z_{n}}{\mathbf{E}\left(Z_{n}\right)} \geq \frac{Z_{n_{k}}}{\mathbf{E}\left(Z_{n_{k+1}}\right)}=\frac{Z_{n_{k}}}{\mathbf{E}\left(Z_{n_{k}}\right)} \frac{\mathbf{E}\left(Z_{n_{k}}\right)}{\mathbf{E}\left(Z_{n_{k+1}}\right)} \geq \frac{Z_{n_{k}}}{\mathbf{E}\left(Z_{n_{k}}\right)} \frac{k^{2}}{(k+2)^{2}}
$$

As a direct consequence of (12), (13) and (14) we get that

$$
\frac{Z_{n}}{\mathbf{E}\left(Z_{n}\right)} \rightarrow 1, \quad \mu \text {-almost everywhere }
$$


as $n \rightarrow \infty$. The quantitative version follows now from the fact that

$$
\frac{\#\left\{i \leq n: d\left(T^{i}(x), x_{0}\right) \leq r_{i}\right\}}{\sum_{j=1}^{n} \mu\left(B\left(x_{0}, r_{j}\right)\right)}=\frac{Z_{n}(x)}{\mathbf{E}\left(Z_{n}\right)} .
$$

The full statement of Corollary 1 is

Corollary 3. Let $(X, d, \mathcal{A}, \mu, T)$ be a dynamical metric system. For any $x_{0} \in X$, we have:

(i) If $\alpha<\underline{d}_{\mu}\left(x_{0}\right)$, then

$$
\liminf _{n \rightarrow \infty} n^{1 / \alpha} d\left(T^{n}(x), x_{0}\right)=\infty, \quad \text { for } \mu \text {-almost every } x \in X .
$$

(ii) If $T$ is uniformly mixing at $x_{0}$ and $\alpha>\bar{d}_{\mu}\left(x_{0}\right)$, then

$$
\liminf _{n \rightarrow \infty} n^{1 / \alpha} d\left(T^{n}(x), x_{0}\right)=0, \quad \text { for } \mu \text {-almost every } x \in X .
$$

Proof. The condition $\beta<\underline{d}_{\mu}\left(x_{0}\right)$ implies that for $r$ small enough.

$$
\mu\left(B\left(x_{0}, r\right)\right) \leq r^{\beta} .
$$

Therefore, if we take $r_{n}=n^{-(1+\varepsilon) / \beta}$, we have that

$$
\sum_{n} \mu\left(B\left(x_{0}, r_{n}\right)\right) \leq C \sum_{n} r_{n}^{\beta}<\infty .
$$

Then from Corollary 2 we obtain that

$$
\liminf _{n \rightarrow \infty} n^{(1+\varepsilon) / \beta} d\left(T^{n}(x), x_{0}\right) \geq 1
$$

for $\mu$-almost every $x \in X$, for all $\varepsilon>0$ and for all $\beta<\underline{d}_{\mu}\left(x_{0}\right)$.

Now, if $\alpha<\underline{d}_{\mu}\left(x_{0}\right)$, then there exists $\varepsilon>0$ small enough so that also $\beta=(1+2 \varepsilon) \alpha<$ $\underline{d}_{\mu}\left(x_{0}\right)$. Therefore, using (15) we get that

$$
\liminf _{n \rightarrow \infty} n^{1 / \alpha} d\left(T^{n}(x), x_{0}\right)=\liminf _{n \rightarrow \infty} n^{\varepsilon / \beta} n^{(1+\varepsilon) / \beta} d\left(T^{n}(x), x_{0}\right)=\infty,
$$

for $x$ in a set of full $\mu$-measure.

To prove part (ii) observe first that the condition $\beta>\bar{d}_{\mu}\left(x_{0}\right)$ implies that for $r$ small enough

$$
\mu\left(B\left(x_{0}, r\right)\right) \geq r^{\beta},
$$

If we take $r_{n}=n^{-1 / \beta}$ we have that

$$
\sum_{n} \mu\left(B\left(x_{0}, r_{n}\right)\right) \geq C \sum_{n} r_{n}^{\beta}=\infty .
$$

and from Theorem 1 we deduce that, for all $\beta>\bar{d}_{\mu}\left(x_{0}\right)$,

$$
\liminf _{n \rightarrow \infty} n^{1 / \beta} d\left(T^{n}(x), x_{0}\right) \leq 1
$$

for $x$ in a set of full $\mu$-measure.

Now, if $\alpha>\bar{d}_{\mu}\left(x_{0}\right)$, then there exists $\varepsilon>0$ small enough so that also $\beta=(1-\varepsilon) \alpha>$ $\bar{d}_{\mu}\left(x_{0}\right)$. Therefore, using (16) we get that

$$
\liminf _{n \rightarrow \infty} n^{1 / \alpha} d\left(T^{n}(x), x_{0}\right)=\liminf _{n \rightarrow \infty} n^{-\varepsilon / \beta} n^{1 / \beta} d\left(T^{n}(x), x_{0}\right)=0,
$$

for $x$ in a set of full $\mu$-measure. 
Remark 1. It is easy to see that this kind of results can be generalized to obtain results about approximation to several points. For example, if $T$ is uniformly mixing at all the points in a sequence $\left\{x_{i}\right\} \subset X$ then, for $\alpha>\max \left\{\bar{d}_{\mu}\left(x_{i}\right): i=1,2, \ldots\right\}$,

$$
\liminf _{n \rightarrow \infty} n^{1 / \alpha} d\left(T^{n}(x), x_{i}\right)=0, \quad \text { for } i=1,2, \ldots
$$

for $\mu$-almost every $x \in X$.

\section{Inner functions}

As we have mentioned in the introduction, we can think of the extension $f^{*}$ of an inner function with $f(0)=0$ as a metrical dynamical system by taking $d$ as the angular distance in $\partial \mathbf{D}$ and $\mu$ as the Lebesgue measure. Theorem E tells us that $f^{*}$ is uniformly mixing at any point $\xi_{0} \in \partial \mathbf{D}$ with respect to a function of the form $\Phi(x)=K e^{-\alpha x}$. Therefore, Theorem 2 which we stated in the introduction is a consequence of Theorem 1.

In the case that the inner function $f$ has a fixed point $p \in \mathbf{D}$, then by conjugation with an appropriate Möbius transformation $S$ we get a new inner function $g=S^{-1} \circ f \circ S$ with $g(0)=0$. By applying Theorem 2 we obtain easily that if $\sum_{n=1}^{\infty} r_{n}=\infty$ then

$$
\liminf _{n \rightarrow \infty} \frac{d\left(\left(f^{*}\right)^{n}(\xi), \xi_{0}\right)}{r_{n}} \leq C(p), \quad \text { for almost every } \xi \in \partial \mathbf{D}
$$

with $C(p)$ a positive constant. However, more is true because in fact we can put $C(p)=1$ in the above inequality. To see this, let us observe first that an easy modification of the proof of Theorem D gives that $f^{*}$ preserves the harmonic measure $\omega_{p}$. We recall that $\omega_{p}$ can be defined as the unique probability measure such that, for all continuous function $\phi: \partial \mathbf{D} \longrightarrow \mathbf{R}$,

$$
\int_{\partial \mathbf{D}} \phi d \omega_{p}=\widetilde{\phi}(p),
$$

where $\widetilde{\phi}$ is the unique extension of $\phi$ which is continuous in $\overline{\mathbf{D}}$ and harmonic in $\mathbf{D}$. It follows that if $A$ is an $\operatorname{arc}$ in $\partial \mathbf{D}$, then $\omega_{p}(A)$ is the value at the point $p$ of the harmonic function whose radial limits take the value 1 on $A$ and the value 0 on the exterior of $A$.

We will see that the dynamical system $\left(\partial \mathbf{D}, d, \mathcal{B}, \omega_{p}, f^{*}\right)$ is also uniformly mixing at any point $\xi_{0} \in \partial \mathbf{D}$ with respect to $\Phi(x)=K e^{-\alpha x}$. As a consequence we get the following result.

Theorem 3. Let $f: \mathbf{D} \longrightarrow \mathbf{D}$ be an inner function with a fixed point $p \in \mathbf{D}$, but not an automorphism which is conjugated to a rotation. Let also $\xi_{0}$ be any point in $\partial \mathbf{D}$ and let $\left\{r_{n}\right\}$ be a decreasing sequence of positive numbers. If $\sum_{n=1}^{\infty} r_{n}=\infty$, then

$$
\lim _{N \rightarrow \infty} \frac{\#\left\{n \leq N: d\left(\left(f^{*}\right)^{n}(\xi), \xi_{0}\right)<r_{n}\right\}}{\sum_{n=1}^{N} r_{n}}=1, \quad \text { for almost every } \xi \in \partial \mathbf{D} .
$$

In particular,

$$
\liminf _{n \rightarrow \infty} \frac{d\left(\left(f^{*}\right)^{n}(\xi), \xi_{0}\right)}{r_{n}} \leq 1, \quad \text { for almost every } \xi \in \partial \mathbf{D}
$$


We recall that, by the Denjoy-Wolff theorem [D], for any holomorphic function $f$ : $\mathbf{D} \longrightarrow \mathbf{D}$ which is not conjugated to a rotation, there exists a point $p \in \overline{\mathbf{D}}$, the so called Denjoy-Wolff point of $f$, such the iterates $f^{n}$ converge to $p$ uniformly on compact subsets of $\mathbf{D}$. Also, if $p \in \mathbf{D}$ then $f(p)=p$ and if $p \in \partial \mathbf{D}$ then $f^{*}(p)=p$.

Hence, if $f$ is an inner function which is not conjugated to a rotation and does not have a fixed point $p \in \mathbf{D}$ then its Denjoy-Wolff point $p$ belongs to $\partial \mathbf{D}$ and $f^{n}$ converges to $p$ uniformly on compact subsets of $D$. As an example, consider the singular inner function $f(z)=e^{-(1-z) /(1+z)}$ whose Denjoy-Wolff point is $p=1$. Of course $f^{n} \rightarrow 1$ uniformly on compact subsets of $\mathbf{D}$, but it is not difficult to see that also $\left(f^{*}\right)^{n} \rightarrow 1$ for almost every point in $\partial \mathbf{D}$. Recently Bourdon, Matache and Shapiro [BMS] and Poggi-Corradini $[\mathrm{PC}]$ have proved independently that if $f$ is inner with a fixed point in $p \in \partial \mathbf{D}$, then $\left(f^{*}\right)^{n}$ can converge to $p$ for almost every point in $\partial \mathbf{D}$. In fact, see [BMS, Theorem 4.2], $\left(f^{*}\right)^{n} \rightarrow p$ almost everywhere in $\partial \mathbf{D}$ if and only if $\sum_{n}\left(1-\left|f^{n}(0)\right|\right)<\infty$.

Proof of Theorem 3. Let $S$ be a Möbius transformation $S: \mathbf{D} \longrightarrow \mathbf{D}$ such that $S(0)=p$. It is easy to check that

$$
\omega_{p}(A)=m\left(S^{-1}(A)\right), \quad \text { for any Borel set } A \subset \partial \mathbf{D} .
$$

We also have that $g=S^{-1} \circ f \circ S$ is inner and $g(0)=0$. If $A, B \subset \partial \mathbf{D}$ are arcs then $A^{\prime}=S^{-1}(A)$ and $B^{\prime}=S^{-1}(B)$ are also arcs in $\partial \mathbf{D}$. By applying Theorem $\mathrm{E}$ to the function $g$ and the $\operatorname{arcs} A^{\prime}, B^{\prime}$ we have that there exist constants $K, \alpha>0$ such that, for all $n \in \mathbf{N}$,

$$
\left|\frac{m\left(B^{\prime} \cap\left(g^{*}\right)^{-n}\left(A^{\prime}\right)\right)}{m\left(A^{\prime}\right)}-m\left(B^{\prime}\right)\right| \leq K e^{-\alpha n} .
$$

Since $\left(f^{*}\right)^{n}=S \circ\left(g^{*}\right)^{n} \circ S^{-1}$, we obtain from (18) and (19) that

$$
\left|\frac{\omega_{p}\left(B \cap\left(f^{*}\right)^{-n}(A)\right)}{\omega_{p}(A)}-\omega_{p}(B)\right| \leq K e^{-\alpha n},
$$

for for all $n \in \mathbf{N}$. Therefore, $f^{*}$ is uniformly mixing at any point with respect to $\omega_{p}$ and $\Phi(x)=K e^{-\alpha x}$. Since $\omega_{p}$ and Lebesgue measure have the same null sets the result follows now from Theorem 1 .

We can rewrite our results for the case of inner functions as follows

Corollary 4. If $f$ is inner with a fixed point $p \in \mathbf{D}$, but not an automorphism which is conjugated to a rotation, then, for any point $\xi_{0} \in \partial \mathbf{D}$, we have:

(i) If $\alpha<1$, then

$$
\liminf _{n \rightarrow \infty} n^{1 / \alpha} d\left(\left(f^{*}\right)^{n}(\xi), \xi_{0}\right)=\infty, \quad \text { for almost every } \xi \in \partial \mathbf{D} .
$$

(ii) If $\alpha>1$, then

$$
\liminf _{n \rightarrow \infty} n^{1 / \alpha} d\left(\left(f^{*}\right)^{n}(\xi), \xi_{0}\right)=0,, \quad \text { for almost every } \xi \in \partial \mathbf{D} .
$$

(iii) In the "critical exponent" $\alpha=1$, we have

$$
\liminf _{n \rightarrow \infty}(n \log n) d\left(\left(f^{*}\right)^{n}(\xi), \xi_{0}\right) \leq 1, \quad \text { for almost every } \xi \in \partial \mathbf{D} .
$$

Proof. Parts (i) and (ii) follow from Theorem 3 reasoning as in Corollary 3. Part (iii) follows directly from Theorem 3 using the sequence of radii $r_{n}=1 /(n \log n)$. 


\section{$5 \quad$ An application: The maps $x \mapsto m x(\bmod 1)$}

We are going to apply our results to expansions in base $m$ of numbers in the interval $[0,1]$. Let us consider the map $T(x)=m x(\bmod 1)$ with $m \in \mathbf{N}, m \geq 2$ acting in $[0,1]$. If $x \in(0,1)$ then $x$ have a unique representation of the type

$$
x=\frac{a_{1}}{m}+\frac{a_{2}}{m^{2}}+\cdots+\frac{a_{k}}{m^{k}}+\cdots, \quad \text { with } a_{i} \in \mathbf{N}, 0 \leq i \leq m-1,
$$

if we do not permit representations where $a_{j}=m-1$ for all $j \geq j_{0}$, for some $j_{0} \in \mathbf{N}$. This is the base $m$ representation of the number $x$. We will write $x$ as

$$
x=\left[\begin{array}{lllll}
a_{1} & a_{2} & \ldots & a_{k} & \ldots
\end{array}\right] .
$$

Observe that

$$
x=\left[\begin{array}{lllll}
a_{1} & a_{2} & \ldots & a_{k} & \ldots
\end{array}\right] \Longrightarrow T(x)=\left[\begin{array}{lllll}
a_{2} & a_{3} & \ldots & a_{k} & \ldots
\end{array}\right],
$$

i.e. $T$ acts as a shift if we represent the numbers $x \in[0,1]$ in base $m$.

Using our results we obtain the following consequence which tells us, in particular that almost every point in $[0,1]$ has arbitrarily long sequences of consecutive zeroes in its base $m$ representation.

Theorem 4. (i) For almost all $x \in[0,1], x=\left[\begin{array}{lllll}a_{1} & a_{2} & \ldots & a_{k}\end{array}\right]$, we have that for infinitely many $n$,

$$
a_{n+1}=a_{n+2}=\cdots=a_{n+\varphi(n)}=0 \quad \text { with } \varphi(n)=\left[\log _{m} n\right] .
$$

In fact,

$$
\lim _{N \rightarrow \infty} \frac{\#\left\{n \leq N: a_{n+1}=a_{n+2}=\cdots=a_{n+\varphi(n)}=0\right\}}{\log N}=1
$$

for almost all $x \in[0,1]$.

(ii) The set of points $x \in[0,1], x=\left[\begin{array}{lllll}a_{1} & a_{2} & \ldots & a_{k} & \ldots\end{array}\right]$, such that for infinitely many $n$,

$$
a_{n+1}=a_{n+2}=\cdots=a_{n+\varphi(n)}=0 \quad \text { with } \varphi(n)=n
$$

has zero Lebesgue measure.

Here $[x]$ means the integer part of $x$. Let us observe that we have chosen the function $\varphi(n)=\left[\log _{m} n\right]$ in part (i) in order that $\sum_{n=1}^{\infty} 1 / m^{\varphi(n)}=\infty$. Similarly the election of $\varphi(n)=n$ in part (ii) gives $\sum_{n=1}^{\infty} 1 / m^{\varphi(n)}<\infty$.

Proof. If $x=\left[\begin{array}{lllll}a_{1} & a_{2} & \ldots & a_{k} & \ldots\end{array}\right]$, for any function $\varphi: \mathbf{N} \longrightarrow \mathbf{N}$, we have that

$$
T^{n}(x) \leq \frac{1}{m^{\varphi(n)+1}} \Longrightarrow a_{n+1}=a_{n+2}=\cdots=a_{n+\varphi(n)}=0,
$$

and therefore the statement

$$
T^{n}(x) \leq \frac{1}{m^{\varphi(n)+1}}, \quad \text { for infinitely many } n,
$$

means that the sequences

$$
a_{n+1}=0, a_{n+2}=0, \ldots, a_{n+\varphi(n)}=0
$$


appear in the base $m$ representation of $x$ for infinitely many $n$.

Let us observe also that $\left([0,1], d_{\text {euclidean }}, \mathcal{B},|\cdot|, T\right)$ is a dynamical system which is isomorphic to $(\partial \mathbf{D}, d, \mathcal{B},|\cdot|, f)$ with $f$ the inner function $f(z)=z^{m}$.

By taking $x_{0}=0 \in[0,1]$, part (i) follows now from Theorem 2 by taking $r_{n}=$ $1 / m^{\left[\log _{m} n\right]+1}$ and using that if $\varphi(n)=\left[\log _{m} n\right]$, then

$$
\sum_{n=1}^{N} \frac{1}{m^{\varphi(n)+1}} \asymp \log N, \quad \text { as } N \rightarrow \infty .
$$

Part (ii) follows from Proposition 1 if we take $r_{n}=1 / m^{n+1}$.

We have made the reduction to the point $x_{0}=0 \in[0,1]$ in order to get a better understanding of the result. One can prove the same result for any point $x_{0}$ in $[0,1]$, for example, by composing the inner function $z^{m}$ with any rotation. Thus, the full statement of the above theorem is the following

Theorem 5. Let $x_{0}=\left[\begin{array}{lllll}b_{1} & b_{2} & \ldots & b_{k} & \ldots\end{array}\right]$ be a point in $[0,1)$. Then,

(i) For almost all $x \in[0,1], x=\left[\begin{array}{lllll}a_{1} & a_{2} & \ldots & a_{k} & \ldots\end{array}\right]$, we have that for infinitely many $n$,

$$
a_{n+1}=b_{1}, a_{n+2}=b_{2}, \ldots, a_{n+\varphi(n)}=b_{\varphi(n)} \quad \text { with } \varphi(n)=\left[\log _{m} n\right] .
$$

In fact,

$$
\lim _{N \rightarrow \infty} \frac{\#\left\{n \leq N: a_{n+1}=b_{1}, a_{n+2}=b_{2}, \ldots, a_{n+\varphi(n)}=b_{\varphi(n)}\right\}}{\log N}=1,
$$

for almost all $x \in[0,1]$.

(ii) The set of points $x \in[0,1], x=\left[\begin{array}{lllll}a_{1} & a_{2} & \ldots & a_{k} & \ldots\end{array}\right]$, such that for infinitely many $n$,

$$
a_{n+1}=b_{1}, a_{n+2}=b_{2}, \ldots, a_{n+\varphi(n)}=b_{\varphi(n)} \quad \text { with } \varphi(n)=n
$$

has zero Lebesgue measure.

\section{References}

[A] Aaronson, J. Ergodic theory of inner functions of the upper half plane. Ann. Henri Poincaré 14 (1978), 233-253.

[ACS] Afraimovich, V., Chazottes, J.R., Saussol, B. Pointwise dimensions for Poincaré recurrence associated with maps and special flows. Disc. Cont. Dyn. Syst. A 9 (2003), 263-280.

[B] Boshernitzan, M. Quantitative recurrence results. Invent. Math. 113 (1993), 617-631. [BGI] Bonanno, C., Galatolo, S., Isola S. Recurrence and algorithmic information. Nonlinearity, 17 (2004), 1057-1074.

[BMS] Bourdon, P.S., Matache, V., Shapiro, J.H., On convergence to the Denjoy-Wolff point. Preprint, 2004.

[BS] Barreira, L., Saussol, B. Hausdorff dimension of measures via Poincaré Recurrence. Comm. Math. Phys. 219 (2001), 443-463.

[CFS] Cornfeld, I.P., Fomin, S.V., Sinai, Ya, G., Ergodic Theory. Springer-Verlag, 1982.

[C1] Craizer, M., Entropy of inner functions. Israel J. Math. 74 (1991), 129-168. 
[C2] Craizer, M., The Bernoulli property of inner functions. Ergod. Th. $\mathscr{E}$ Dynam. Sys. 12 (1992), 209-215.

[D] Denjoy, A., Fonctions contractante le cercle $|z|<1$. C.R. Acad. Sci. Paris 182 (1926), 255-257.

[F] Furstenberg, H. Recurrence in Ergodic Theory and Combinatorial Number Theory. Princeton University Press, 1981.

[FP] Fernández, J.L., Pestana, D., Distortion of Boundary Sets under Inner Functions and Applications. Indiana U. Math J. 41 (1992), 439-448.

[FPR] Fernández, J.L., Pestana, D., Rodríguez, J.M., Distortion of Boundary Sets under Inner Functions II. Pacific J. Math. 172 (1996), 49-81.

[G] Galatolo, S. Dimension via waiting time and recurrence. Math. Research Letters, 12 (2005), 377-386.

$[\mathrm{K}]$ Kleinbock, D., Metric Diophantine Approximation and dymamical systems. Brandeis University Notes (http://people.brandeis.edu/ kleinboc/203b/lectures.pdf).

[M] Mañé. R. Ergodic Theory and Differentiable Dynamics. Springer-Verlag, 1987.

[N] Neuwirth, J.H. Ergodicity of some mappings of the circle and the line. Israel J. Math. 31 (1978), 359-367.

[P] Pommerenke, Ch. On ergodic properties of inner functions. Math. Ann. 256 (1981), 43-50.

[PC] Poggi-Corradini, P., Pointwise convergence on the boundary in the Denjoy-Wolff theorem. Preprint, 2005.

[PR] Pestana, D., Rodríguez, J.M., Capacity distortion by inner functions in the unit ball of $\mathbf{C}^{n}$. Michigan Math. J. 44 (1997), 125-137.

[R] Rudin, W. Function theory in the unit ball of $\mathbf{C}^{n}$. Springer-Verlag, 1980.

[STV] Saussol, B. Troubetzkoy, S., Vaienti, S. Recurrence, dimensions and Lyapunov exponents. J. Stat. Phys. 106 (2002), 623-634.

[S] Sullivan, D., Disjoint spheres, approximation by imaginary quadratic numbers and the logarithm law for geodesics Acta Math. 149 (1982), 215-237.

[W] Walters P. An introduction to Ergodic Theory. Springer- Verlag, 1981. 